Nils Reiter, Marcus Willand und Evelyn Gius

\title{
Die Erstellung von Annotationsrichtlinien als Community-Aufgabe für die Digitalen Geisteswissenschaften
}

\section{Ein Vorschlag zu Shared Tasks in den Digital Humanities}

\begin{abstract}
The article describes the idea, operationalization decisions and results of the first shared task in the Digital Humanities. In the task, different participating teams developed annotation guidelines for narrative levels independently. Annotation guidelines are a prerequisite for the development of systems for the automatic detection of textual phenomena, and thus needed in Computational Literary Studies because they allow large-scale studies of narrative phenomena. The developed guidelines were compared using a newly developed evaluation scheme that brings together the three dimensions of conceptual coverage, applicability and usefulness.
\end{abstract}

Keywords: Annotation Template, Evaluation, Literature, Methodology, Narratology

\section{Einleitung}

Annotationsrichtlinien für literarische Phänomene sind ein zentrales Desiderat im Bereich der textorientierten digitalen Geisteswissenschaften. Die Erstellung von allgemein anwendbaren Richtlinien ist jedoch nur in großen und dementsprechend ressourcenintensiven Annotationsprojekten möglich. Darüber hinaus sind einerseits Wissenschaftler*innen, die an umfangreichen Analysen literarischer Texte interessiert sind, gefordert, viele Aufgaben zu erfüllen, die außerhalb ihrer Kernkompetenz liegen, während andererseits Forscher`innen aus der Informatik, die sich für die Methodenentwicklung interessieren, selbst annotierte Daten erstellen müssen. Shared Tasks - ein Forschungsformat, das in der maschinellen Sprachverarbeitung verbreitet ist - bieten einen Lösungsvorschlag für die genannten Problembereiche an.

Dieser Beitrag fasst das Konzept und die Ergebnisse des ersten Shared Tasks in den digitalen Geisteswissenschaften zusammen. Dieser Shared Task begann

\footnotetext{
Nils Reiter, Institut für Maschinelle Sprachverarbeitung, Universität Stuttgart Marcus Willand, Germanistisches Seminar, Universität Heidelberg Evelyn Gius, Institut für Sprach- und Literaturwissenschaft, Technische Universität Darmstadt
} 
im Mai 2018 und ist der erste, der die Entwicklung von Annotationsrichtlinien als Hauptziel hat. ${ }^{1}$

Er befasst sich im Kern mit zwei Aspekten, die in den digitalen Geisteswissenschaften und der computergestützten Literaturwissenschaft wesentlich sind: Mit der Aufteilung von Arbeit, Kompetenzen und Aufgaben im interdisziplinären Forschungsfeld der digitalen Geisteswissenschaften und mit der intersubjektiven manuellen und zuverlässigen automatischen Erkennung von Erzählebenen in Erzähltexten.

\subsection{Aufteilung von Arbeit, Aufgaben und Kompetenzen}

Angesichts des aktuellen Stands der rechnergestützten Analyse von narrativen Texten $^{2}$ müssen digitale geisteswissenschaftliche Projekte, die darauf abzielen, inhaltliche Aspekte solcher Texte in großem Maßstab zu analysieren, selbst technischmethodische Entwicklungen liefern, um die gewünschten Phänomene automatisch zu erkennen. Viele dieser Projekte sind daher Kooperationsprojekte zwischen Forscher`innen aus den Bereichen Informatik/Natural Language Processing (NLP) und Literatur- oder Kulturwissenschaften. Obwohl es eine wachsende Anzahl von Tutorials, How-Tos und Lehrbüchern zu verschiedenen Themen der digitalen Geisteswissenschaften gibt, ${ }^{3}$ bleibt die Bearbeitung solcher digitalen geisteswissenschaftlichen Projekte aus verschiedenen Gründen schwierig:

(i) Die Entwicklung einer gemeinsamen Sprache, bzw. Terminologie und eines gemeinsamen Verständnisses des oft komplexen Forschungsproblems ist eine der ersten Hürden, die digitale geisteswissenschaftliche Projekte überwinden müssen.

(ii) Während sich Informatikerinnen typischerweise nur für den methodischen Teil interessieren und eine Interpretation der Ergebnisse in Bezug auf die zu untersuchenden Texte außer Acht lassen, konzentrieren sich Geisteswissenschaftler^innen typischerweise auf konzeptionelle Fragen oder Fragen der Ergebnisinterpretation.

1 Dieser Beitrag basiert im Wesentlichen auf einer zusammenfassenden Darstellung der Special Issue von Cultural Analytics, die neben einer ausführlichen Diskussion des Zugangs auch die eingereichten Guidelines und ihre Gutachten umfasst: Gius et al. (2019)

2 Die Performance von NLP-tools ist bei der Analyse narrativer Texte aufgrund deren Eigenschaften wie Literarizität und Poetizität üblicherweise weniger gut als es der Stand der Technik für nichtliterarische Texte ermöglicht: https://nlpprogress.com (25.12.2019).

3 Unter anderem vgl. Schreibman et al. (2004); Siemens und Schreibman (2008); Jockers (2014); Jannidis et al. (2017); Silva Knuppel und Afanador-Llach (2019). 
(iii) Daher können die Ziele der Partner*innen auch innerhalb desselben Projekts unterschiedlich sein. Wir glauben, dass Formate wie dieser Shared Task großes Potential für die digitalen Geisteswissenschaften haben, denn sie erlauben es, dass sich die Teilnehmer*innen auf genau das konzentrieren können, worin sie geschult wurden: Literaturwissenschaftler^innen können sich auf jene literarischen Phänomene konzentrieren, die sie interessieren und für deren Analyse sie über Expertise verfügen. Mit ihren disziplinären Routinen und Textzugängen sind Literaturwissenschaftler ${ }^{\star}$ innen am besten qualifiziert, narratologische Konzepte zu erforschen, zu (re-)definieren und zu veranschaulichen. In einem Shared Task nach dem von uns vorgeschlagenen Modell können sie dies tun, ohne sich um die Automatisierbarkeit ihrer Ergebnisse zu kümmern. Die da mit dem Automatisierungsanspruch üblicherweise einhergehende Simplifizierung literaturwissenschaftlicher Konzepte ist längerfristig gesehen eine Sackgasse für methodische Innovationen, da sich auch die Einsatzmöglichkeiten von Computern ständig weiterentwickeln.

Die zentrale Voraussetzung für literaturwissenschaftlich anschlussfähige Analysen ist jedoch, dass die konzeptionelle Komplexität in intersubjektiv anwendbaren Annotationsrichtlinien abgebildet wird und ein Korpus auf Grundlage dieser Richtlinien annotiert wurde. Dann können Informatiker*innen oder andere Expertinnen für maschinelles Lernen an der automatischen Erkennung der Konzepte arbeiten. Literaturwissenschaftliche Expertise ist dafür nicht nötig, weil die Annotationen konzeptionelle literaturwissenschaftliche Entscheidungen beinhalten (ähnlich wie das bei Standardkorpora aus der natürlichen Sprachverarbeitung der Fall ist).

Bei der Anwendung von maschinellem Lernen in einem digitalen geisteswissenschaftlichen Szenario gibt es oft einen Kompromiss zwischen Leistung und Transparenz: Modelle, die eine bessere Leistung erzielen (z. B. neuronale Netze), sind oft weniger transparent, während transparente Modelle (z. B. Entscheidungsbäume) typischerweise geringere Leistung erzielen. In diesem Fall können sich Informatiker*innen aufgrund der empirischen Auswertung für das performanteste Modell entscheiden. Um von der Expertise beider beteiligten Felder in der skizzierten Weise zu profitieren, organisieren wir zwei Shared Tasks, die diese zwei Seiten von Annotation - manuelle und automatische Erzeugung - abbilden. Der erste Shared Task bildet die Grundlage für eine unabhängige und zuverlässige empirische Bewertung der späteren automatischen Erkennungssysteme. So kann ein Modell, das im zweiten Shared Task gut funktioniert hat, für neue Texte verwendet werden, die ähnlich wie die Testdaten sind (was für Literaturwissenschaftler^innen wiederum transparent ist).

Die Entkopplung der Konzept- von der Implementierungsarbeit ermöglicht, dass sich jede`r auf das eigene Fachgebiet konzentrieren kann: Literaturwissen- 
schaftler^innen können sich auf die Entwicklung von Annotationsrichtlinien konzentrieren. Dazu gehören konzeptionelle Arbeiten ebenso wie ein erster Schritt zur Operationalisierung wissenschaftlicher Konzepte (soweit sie intersubjektiv anwendbar sind). Bei dieser Arbeitsweise müssen die Literaturwissenschaftler`innen nicht im selben Projekt, an derselben Universität oder sogar auf demselben Kontinent sein wie die Forscher^innen, die die automatischen Erkennungswerkzeuge entwickeln. Dadurch werden die Eintrittsbarrieren in die computationelle Literaturwissenschaft bzw. die Digital Humanities gesenkt, da man nicht in einem gut finanzierten interdisziplinären Projekt arbeiten muss, um zu den übergeordneten Zielen beizutragen. Stattdessen können Wissenschaftler*innen in ihrem eigenen Tempo und innerhalb ihres disziplinären Paradigmas arbeiten und ihren Beitrag leichter in ihre eigene Forschungsagenda integrieren. Darüber hinaus ist so eine Zusammenarbeit möglich, die weitgehend ohne die in interdisziplinären Kooperationen übliche erhöhte Arbeitsbelastung auskommt.

\subsection{Erzählebenen als gemeinsames Phänomen der Shared Tasks}

In unserem Vorhaben beschäftigen sich die Teilnehmer`innen mit Erzählebenen. Die Erkennung von Erzählebenen und damit die Identifizierung von kohärenten Textteilen ist für die Analyse von Erzähltexten erforderlich, um eine anschließende, inhaltsbezogene literaturwissenschaftliche Forschung auf der Grundlage der gewonnenen Daten (über Handlung, Figuren, erzählte Welt, etc.) zu ermöglichen. Gleichzeitig sind Erzählebenen ein derart ubiquitäres Phänomen, dass sie sehr oft nicht einmal in der Literaturwissenschaft explizit adressiert werden. Die automatische Erkennung von Erzählebenen ist daher ein entscheidender Beitrag im Bereich der computergestützten Literaturwissenschaft und als Grundlagenforschung zu versehen. Darüber hinaus können narrative Ebenen ein Mittler sein, der hermeneutische und automatische Textanalyse verbindet. Auch wenn die Komplexität der Erzählebenen aus literaturwissenschaftlicher Sicht als vergleichsweise gering und aus Sicht der natürlichen Sprachverarbeitung als vergleichsweise hoch angesehen wird, ist sie für Textanalysen aller Art relevant und damit ein guter Bezugspunkt für beide Seiten. Außerdem sind Erzählebenen im Vergleich zu anderen Phänomenen ein eher wenig umstrittenes Phänomen in der Literaturwissenschaft. Schließlich basieren die Definitionen von Erzählebenen in der Regel auf Textmerkmalen. So können beispielsweise Verben der Äußerung und der anschließenden direkten Rede textuelle Signale für narrative Ebenen sein, ebenso wie das Vorhandensein einer anderen fiktionalen Welt, die wiederum durch die Analyse des Raumes oder anderer narrativer Phänomene identifiziert werden kann. Narrative Ebenen sind 
daher für die Analyse von Texten hilfreich, die eine Abweichung zwischen ihrer textlichen Struktur und der Struktur des Erzählten aufweisen.

Wir betrachten narrative Ebenen als eine gute Grundlage für einen Shared Task. Ihre wichtigste Eigenschaft für unsere Zwecke ist, dass sie die Lücke zwischen der theoretischen Diskussion eines Phänomens und der Anwendung im Text Mining schließen können. Um den wissenschaftlichen Nutzen der daraus resultierenden automatischen Erkennungsinstrumente zu gewährleisten, müssen narratologische Expert*innen in den Prozess der Richtlinienerstellung einbezogen werden.

Im verbleibenden Beitrag stellen wir anhand der Konzeption und Durchführung des ersten Shared Tasks zur Erstellung von Annotationsrichtlinien für Erzählebenen dar, wie dies geschehen kann. Auf Grundlage der Ergebnisse des Shared Tasks zur Erstellung von Annotationsrichtlinien wird der noch anstehende zweite Shared Task zur Automatisierung der Erkennung von Erzählebenen organisiert werden.

\section{Annotationen}

Der Begriff ,Annotation“ wird innerhalb der DH-Community mit unterschiedlichen Bedeutungen verwendet. In unserem Projekt wird der Begriff für den Prozess verwendet, bei dem Segmente eines Textes als zu einer definierten Kategorie gehörig markiert werden. Wir gehen auch davon aus, dass solche Kategorien vorher festgelegt werden (womit wir rein explorative oder erläuternde Annotationen ausschließen) und dass ihre Erkennung auf dem Inhalt des Textes und nicht auf Struktur oder Formatierung basiert (womit die Annotation von Textstrukturen wie in TEI $\mathrm{XML}^{4}$ nicht im Fokus steht). Dies bedeutet auch, dass das Erkennen dieser Kategorien nicht trivial ist und ein Textverständnis und ein Maß an Textinterpretation erfordert.

Dieses Verständnis der Annotation ist dem linguistischen Begriff der Annotation von z. B. Koreferenzketten oder semantischen Rollen am ähnlichsten. ${ }^{5}$ Es gibt jedoch eine Reihe von Eigenschaften von Annotationen narrativer Phänomene, die typisch für die literaturwissenschaftliche Textanalyse sind und für den

$4 \mathrm{http}: / /$ www.tei-c.org (16.01.2020)

5 Die Annotation von Koreferenzketten ist die Aufgabe, festzustellen, welche Erwähnungen einer Entität sich auf dieselbe beziehen (z. B. in „Ein Haus wurde von Maria gekauft. Petrus liebt sie“, das Pronomen „sie“ bezieht sich auf Maria). Die Identifizierung semantischer Rollen würde uns sagen, dass Maria das Agens des ersten Satzes ist, und „ein Haus“ das Patiens oder das Thema (d. h. das, was gekauft wurde). 


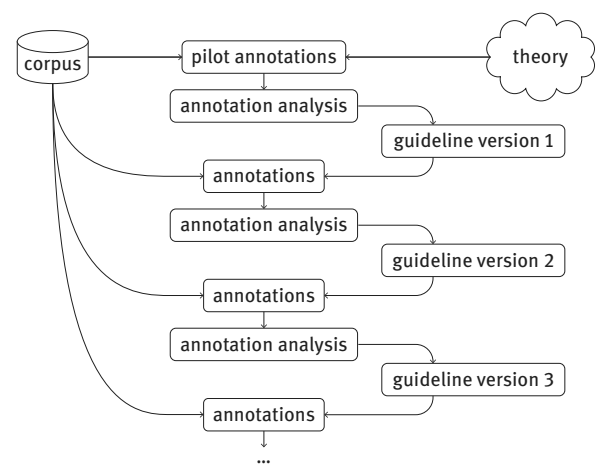

Abb. 1: Iterativer Annotations-Workflow

Annotations-Workflow berücksichtigt werden müssen. So muss etwa ein Phänomen wie Erzählebenen möglicherweise in einem großen Teil des gesamten Textes annotiert werden, während typische linguistische Phänomene wie semantische Rollen auf einzelne Wörter oder Phrasen beschränkt sind. Darüber hinaus ist der für die Bestimmung relevante Kontext in der Regel viel größer. Für die linguistischen Annotationsaufgaben, die in der Vergangenheit Gegenstand von Shared Tasks waren, genügt oft ein Kontextfenster von einem einzigen Satz. ${ }^{6}$ Literaturwissenschaftliche Annotationen betrachten das gesamte Dokument regelmäßig als relevanten Ko(n)text und erfordern daher umfassende Textkenntnis der Annotator*innen. Es ist durchaus denkbar (aber nicht einfach umzusetzen), auch text-externe Quellen (z. B. sozio-historische Umstände) als relevanten Kontext zu betrachten. Dieser größere Kontext hat das Potenzial, literaturwissenschaftliche Annotationen interpretativer zu machen als linguistische.

\subsection{Der Annotationsprozess}

Der Annotationsprozess in computationellen Zugängen mit literaturwissenschaftlicher Fundierung ist iterativ und eng mit der Entwicklung einer Annotationsrichtlinie verbunden. Dieser iterative Prozess ist in Abbildung 1 dargestellt und ist dem MATTER-Zyklus ähnlich (Stubbs und Pustejovsky 2013). In jedem Prozessschritt erhöht sich nicht nur die Anzahl der annotierten Texte, sondern es wird auch die Annotationsrichtlinie verbessert. Natürlich müssen Änderungen in der Annotationsrichtlinie berücksichtigt werden. Dies kann dazu führen, dass bereits annotierte Textstellen überarbeitet werden müssen. Die Kernidee in diesem An-

6 Die Annotation von Koreferenzketten ist insofern eher untypisch, da sie als Aufgabe auf Dokumentebene betrachtet wird und volle Textkenntnis erfordert. 
notationsprozess - mit dem Ziel, kohärente und intersubjektive Annotationen zu erzeugen - besteht darin, dass mehrere Annotator`innen zumindest für einen Teil der Daten die gleichen Texte parallel annotieren. In der Praxis handelt es sich dabei oft um studentische Hilfskräfte, die von den theoretischen Grundlagen vielleicht nur ein rudimentäres Verständnis haben. Die Parallelannotation ermöglicht die Überprüfung und den Vergleich von Annotationen und damit die Identifizierung von Problemen in der Richtlinie. Dieser Annotations-Workflow weist deutliche Parallelen zum hermeneutischen Zirkel auf, der ein allgemeines epistemologisches Muster in den Geisteswissenschaften beschreibt. Entsprechend hat der Annotations-Workflow (und die iterative Verfeinerung der Annotationsvorgaben) Auswirkungen auf die theoretische Ebene und kann produktiv für die Entwicklung und Verfeinerung theoretischer Konzepte eingesetzt werden. ${ }^{7}$

\subsection{Annotationsrichtlinien}

Um kohärente und systematische Annotationen zu erzeugen, werden Annotationen mit Hilfe von Annotationsrichtlinien durchgeführt. Annotationsrichtlinien vermitteln zwischen einem spezifischen theoretischen Verständnis von Konzepten (wie dem einer narrativen Ebene) und der praktischen Annotation des Konzepts in Texten. Sie haben mehrere Ziele, die alle auf die Erklärung theoretischer Konzepte und/oder den Prozess der Annotation ausgerichtet sind:

1. Lücken füllen: Theorien sind oft nicht spezifisch genug, um direkt angewendet zu werden. Um so abstrakt wie möglich zu sein, vernachlässigen sie in der Regel viele Einzelheiten und sind entsprechend unterdefiniert. Diese Lücken können von Annotator*innen aber während des Annotationsprozesses nicht idiosynkratisch bestimmt werden, sondern müssen durch geeignete Definitionen nachvollziehbar beschrieben und ,gefüllt“" werden. ${ }^{8}$

2. Beispiele geben: Im Idealfall befähigt eine Annotationsrichtlinie auch Annotator^innen, die über keine ausgeprägte literaturwissenschaftliche Expertise verfügen, Annotationen durchzuführen. Zu diesem Zweck werden Beispiele gegeben und/oder Paraphrasen-/Einfügetests formuliert.

3. Textbezogene Anpassungen vornehmen: Selbst bei relativ einfachen linguistischen Phänomenen (z. B. Wortarten) ist nicht zu erwarten, dass die be-

7 Vgl. Gius und Jacke (2017, 233-254) zur hermeneutischen Annäherung an narrative Zeitphänomene und Pagel et al. (2018) für eine allgemeine Workflowbeschreibung.

8 Durch das Füllen der theoretischen Lücken wird zwangsläufig ein Reflexionsprozess in Gang, der natürlich ebenso beabsichtigt ist. Dieser kann als Neben- oder Hauptprodukt gesehen werden, Richtlinien sind aber nicht der Ort dafür um die Ergebnisse davon festzuhalten. 
stehenden Annotationsrichtlinien allumfassend sind, da die Variabilität und Kreativität der menschlichen Sprachproduktion enorm ist und ständig neue Texttypen entstehen. Annotationsrichtlinien sind ein Mittel, um Phänomene anzugehen, die text- oder genrebezogen sind.

4. Als Dokumentation fungieren: Schließlich entsteht in den meisten Annotationsprozessen viel prozedurales Wissen, da Entscheidungen über Grenzfälle täglich getroffen werden müssen. Eine Annotationsrichtlinie dient auch als eine Art Protokoll, das diese Entscheidungen dokumentiert und für andere Forscher*innen nachvollziehbar macht.

\subsection{Bewertung von Annotationen}

Eine möglichst hohe Übereinstimmung zwischen den Annotator`innen ist ein wesentliches Ziel der beschriebenen Art von Annotation: Von zwei Annotator ${ }^{\star}$ innen, die den gleichen Text mit der gleichen Annotationsrichtlinie annotieren, wird im Allgemeinen erwartet, dass sie die gleichen Annotationen erzeugen. ${ }^{9}$ Die Überprüfung von Annotationen im Hinblick auf ihre erzielte Übereinstimmung ist daher ein wesentlicher Bestandteil des Analyseschrittes der Annotationen in Abbildung 1 auf Seite 330.

Die regelmäßige Diskussion von Annotationsentscheidungen mit allen beteiligten Annotator*innen ist eine effektive Möglichkeit, sich über die Schwierigkeiten in der Richtlinie zu informieren. Die Aufforderung an die Annotator`innen, ihre Entscheidungen zu erläutern (insbesondere, wenn sie unterschiedlich oder schwierig waren), fördert nicht nur ihre Aufmerksamkeit und Genauigkeit beim Annotieren, sondern offenbart auch Missverständnisse und/oder zeigt Bereiche auf, in denen die Annotationsrichtlinie verbessert werden kann.

Darüber hinaus kann die Höhe der Übereinstimmung zwischen den Annotator*innen quantifiziert werden. Dies wird als Inter-Annotator-Agreement (IAA) bezeichnet, und es wurden zahlreiche Metriken für verschiedene Arten von Annotationsaufgaben vorgeschlagen (Fleiss 1971, 420-428; Cohen 2017, 37-46; Fournier 2013, 1702-1712; Yann Mathet, Antoine Widlöcher und Jean-Philippe Métivier 2015, 437-479; siehe Artstein und Poesio 2008 für einen Überblick). Alle Metriken zielen darauf ab, ein Gleichgewicht zwischen der beobachteten und erwarteten Übereinstimmung herzustellen. Während die erste ausdrückt, wie gut echte Annotator`innen übereinstimmen, drückt die zweite aus, wie viele Annotationen über-

9 Es gibt Ausnahmen, insbesondere bei literarischen Texten. In diesen Fällen können mehrwertige Textlesungen zu unterschiedlichen Annotationen führen, die einen begründeten Widerspruch darstellen. Vgl. Gius und Jacke (2017, 233-254). 
einstimmend wären, wenn sie zufällig gemacht würden. Somit wird die tatsächlich beobachtete Übereinstimmung in Bezug zur Schwierigkeit der Annotationsaufgabe gesetzt. Der Grund dafür ist, dass es zum Beispiel viel einfacher ist, eine Einigung zu erzielen, wenn es nur zwei Kategorien gibt, als wenn es 25 Kategorien gibt. Somit ist die erwartete Übereinstimmung für zwei Kategorien höher als für 25 Kategorien, was das IAA senkt, wenn die beobachtete Übereinstimmung stabil bleibt. Die meisten IAA-Metriken liegen im Intervall $[-\infty ; 1]$. Werte über Null drücken dabei aus, dass die Annotator*innen mehr als nur zufällig übereinstimmen.

Die Messung des IAA für Aufgaben auf höherer Ebene ist nicht trivial. Dies liegt daran, dass viele dieser Aufgaben tatsächlich aus mehreren Teilaufgaben bestehen und die Annotator ${ }^{\star}$ innen mehrere Entscheidungen nacheinander treffen müssen. Die Annotation von Eigennamen (named entities) erfordert beispielsweise, dass Annotator`innen zuerst ein Segment finden, das ein Eigenname ist, und zweitens dieses Segment einer bestimmten Kategorie zuordnen, wie z. B. Person oder Ort. Die IAA-Metrik muss entweder beide Entscheidungen berücksichtigen, was die genaue Berechnung komplex macht, oder vereinfachende Annahmen verwenden (z. B. um überlappende Spannen zu ignorieren).

In der NLP wird das IAA oft auch als Obergrenze für die Maschinenleistung angesehen. Wenn Menschen nur bis zu einem gewissen Grad übereinstimmen, können wir nicht erwarten, dass Maschinen bessere Ergebnisse erzielen.

\subsection{Das Konzept der Erzählebenen im Shared Task}

Für diesen Shared Task haben wir keinen theoretischen Hintergrund für die Konzepte der narrativen Ebene festgelegt. Stattdessen wurden die Teilnehmerinnen ermutigt, den Ansatz zu wählen, den sie für angemessen hielten. Wir haben auf der Homepage des Shared Tasks eine grundlegende Erklärung sowie Lesevorschläge (kategorisiert als einleitend, grundlegend oder fortgeschritten) gegeben, ${ }^{10}$ aber wir haben absichtlich keinen der Ansätze priorisiert. Daher konnten die Teilnehmer*innen jede (oder sogar keine) narratologische Theorie als Grundlage für die Operationalisierung in ihrem Leitfaden verwenden.

Es gibt mehrere Gründe für diese Entscheidung:

a) Obwohl es in der Narratologie nur wenige etablierte Ansätze für narrative Ebenen gibt und sich die meisten von ihnen überschneiden, gibt es keinen Konsens über das Konzept der narrativen Ebene. Narratolog^innen neigen dazu, starke und unterschiedliche Meinungen über die Art der Erzählebenen

10 Für die vollständige Liste siehe: https://sharedtasksinthedh.github.io/levels/ (16.01.2020) 
$\mathrm{zu}$ haben, und für die meisten verfügbaren Theorien gibt es gute Argumente. Daher gibt es keine a priori Möglichkeit, das am besten geeignete Konzept für die Ebenenannotation auszuwählen.

b) Wie in vielen geisteswissenschaftlichen Disziplinen gibt es kein etabliertes Verfahren zur Identifizierung der ,richtigen‘ Theorie unter den nebeneinander existierenden Ansätzen. Die Vorstellung, dass etwas richtig, wahr, objektiv usw. ist, ist kaum mit dem disziplinären Paradigma oder der Matrix der Geisteswissenschaften vereinbar. Innerhalb des geisteswissenschaftlichen Paradigmas existieren Theorien und Interpretationen typischerweise nebeneinander und können sich sogar widersprechen. Diese Vielfalt ist den Geisteswissenschaften und ihrer oft stark interpretativen Analyse von mehrdeutigen und vielschichtigen menschlichen Artefakten geschuldet. Da der Gesamtprozess des Verstehens komplex ist und seine Teile nicht vollständig verstanden sind, kann die Beschränkung der Analyse eines Artefakts auf die Verwendung bestimmter Theorien zu einem vorzeitigen Ausschluss von Ansätzen führen, die relevante Erkenntnisse liefern. Die Beschränkung der narrativen Ebenenanalyse auf einen Ansatz hätte daher bedeutet, den Prozess zu ignorieren, durch den theoretische oder methodische Ansätze in der Literaturtheorie entwickelt wurden und werden.

c) Annotationsrichtlinien spielen in der zeitgenössischen Narratologie kaum eine Rolle, und die Annotierbarkeit wird derzeit nicht regelmäßig berücksichtigt. Aus narratologischer Sicht ist die reine Richtlinienerstellung wahrscheinlich nicht so interessant wie die Diskussion/der Vergleich von narratologischen Theorien. Von Anfang an war jedoch klar, dass die Beteiligung von Narratologieexpert^innen für diesen Shared Task von größter Bedeutung sein würde. Daher sollte das Einbeziehen verschiedener theoretischer Ausrichtungen auch das Interesse von Narratolog*innen wecken, die neu in der Entwicklung von Annotationen sind.

Vor diesem Hintergrund war die Offenheit im Bezug auf Theorien für den Prozess auf mehreren Ebenen von Vorteil. Am wichtigsten war, dass wir uns an das Paradigma der Geisteswissenschaften halten und gleichzeitig einen Rahmen für die Erforschung und Erprobung von Theorien in diesem ersten Shared Task zur Richtlinienentwicklung schaffen konnten. Dies gewährleistet eine höhere Relevanz der Ergebnisse der Automatisierung für die Anwenderinnen. 


\section{Der Shared Task zur Erstellung von Annotationsrichtlinien}

\subsection{Shared Tasks in der maschinellen Sprachverarbeitung}

Shared Tasks sind ein etabliertes Forschungsformat innerhalb der Natural Language Processing (NLP). Die zentrale Idee ist, dass mehrere Teilnehmer*innen versuchen, die gleiche Aufgabe der Organisator`innen zu lösen (z. B. automatische Vorhersage einer Wortart). Die Lösungen werden dann auf dem gleichen Datensatz mit der gleichen Metrik bewertet und sind damit direkt vergleichbar. Im Allgemeinen funktioniert ein Shared Task wie folgt: Die Organisator*innen veröffentlichen einen Call for participation, der die Aufgabe sowie den zugehörigen Datensatz detailliert beschreibt. Kurz darauf veröffentlichen die Organisator`innen einen Entwicklungs- und/oder Trainingsdatensatz. Der Datensatz enthält so genannte ,Goldinformationen', d. h. die zu identifizierenden Kategorien sind bereits mit Annotationen versehen. Dieser Datensatz wird dann von den Teilnehmer^innen verwendet, um Systeme zu entwickeln/trainieren, die die definierte Aufgabe automatisch lösen. Nach mehreren Monaten Entwicklungszeit veröffentlichen die Organisator`innen einen zweiten Datensatz ohne die Annotationen: die Testdaten. Die Teilnehmer^innen wenden ihre Systeme auf den Testdatensatz an (typischerweise innerhalb einer Woche) und senden die von ihren Systemen gemachten Vorhersagen an die Organisator ${ }^{\star}$ innen. Die Organisator ${ }^{\star}$ innen werten dann die Vorhersagen aller Systeme mit dem gleichen Auswertungsskript und mit den gleichen Referenzdaten aus. Danach kann ein Ranking der Systeme erstellt werden, und es wird ein Workshop durchgeführt, um die verschiedenen Systeme vorzustellen und die Ergebnisse zu diskutieren.

Parra Escartín et al. (1971, 66-73) diskutieren mehrere Gründe für die Beliebtheit und den Erfolg von Shared Tasks in der maschinellen Sprachverarbeitung: Sie fördern nicht nur die Entwicklung in einem bestimmten Bereich, sondern ermöglichen auch den direkten Vergleich zwischen den Systemen. In Shared Tasks haben sich eine Reihe von De-facto-Standards entwickelt (z. B. das weit verbreitete CoNLL-Format zur Speicherung annotierter Daten). Darüber hinaus wurden zusammen mit den Shared Tasks kuratierte Datensätze erstellt und anschließend zur Verfügung gestellt. 


\subsection{Shared Tasks in den Digital Humanities}

Da sich die Forschungspraktiken, Ziele und nicht zuletzt die Forschungsgemeinschaften in der NLP und der Literaturwissenschaft deutlich unterscheiden, wird die direkte Anwendung des NLP-Modells nicht funktionieren. Deshalb haben wir das Verfahren mehrfach angepasst. Die beiden Shared Tasks haben unterschiedliche Ziele, Datensätze und Zielgruppen, konzentrieren sich aber beide auf das Phänomen der narrativen Ebenen. Das Ziel von Task 1 ist die Erstellung von Annotationsrichtlinien, die dann verwendet werden, um ein großes Korpus zu annotieren, das als Trainings-/Testdaten in Task 2 verwendet werden soll. Diese ist ein ,normaler' Shared Task mit dem Ziel, Systeme zu entwickeln, die automatisch narrative Ebenen erkennen.

\subsubsection{Der Shared Task 1: Systematische Analyse von narrativen Texten durch Annotation (SANTA)}

Im ersten Shared Task stehen die Herausforderungen der Konzeption und Definition von Erzählebenen sowie deren manuelle Anwendung auf Texte im Mittelpunkt. Auf unserer Webseite haben wir auch einen „How to“-Artikel veröffentlicht, der den obigen Annotationsworkflow erklärt.

Um die Entwicklung allgemeiner Richtlinien zu fördern, haben wir frühzeitig beschlossen, dass die Richtlinien an einem bis auf das Genre nicht näher spezifizierten Korpus getestet werden sollten. Jede ${ }^{\star}$ T Teilnehmer ${ }^{\star}$ in musste also die Richtlinie schreiben, ohne die genauen Texte zu kennen, auf die sie am Ende angewendet werden würde. Um die Vergleichbarkeit der Richtlinien zu gewährleisten, musste jedoch eine gewisse Homogenität des Korpus erreicht werden. Wir haben den Teilnehmer`innen daher ein Entwicklungskorpus zur Verfügung gestellt, das sie bei der Erstellung der Richtlinien verwenden können. Dieser Aufbau ist inspiriert von der Unterscheidung zwischen Entwicklungs-, Trainings- und Testdaten, die beim maschinellen Lernen verwendet wird (vgl. Witten und Eibe 2005, 144 ff.).

Überlegungen zum Korpus. Das Korpus wurde zusammengestellt, um möglichst viele der vorgeschlagenen Ebenenphänomene abzudecken. Es ist in Bezug auf Genre, Erscheinungsdatum und Textlänge heterogen. ${ }^{11}$ Die Repräsentativität (was auch immer das für die Literatur bedeutet) war jedoch kein Leitprinzip. Alle Texte wurden sowohl auf Englisch als auch auf Deutsch zur Verfügung gestellt, einige

11 Genres: Anekdote, Fabel, Volksmärchen, literarisches Märchen, Roman, Novelle, Erzählung, Kurzgeschichte. Erscheinungsdatum: Die meisten Texte stammen aus dem 19. und 20. Jahrhundert. Textlänge: maximal 2000 Wörter. 
davon sind Übersetzungen aus einer dritten Sprache. Die maximale Länge der Texte im Korpus durfte 2000 Wörter nicht überschreiten. Diese Einschränkung könnte allerdings auch einen Einfluss auf die Verwendung von Erzählebenen haben, was zu einer Verzerrung unseres Datensatzes geführt hätte. Um diese zu vermeiden, haben wir auch ursprünglich längere Texte in gekürzter Form eingefügt. Dabei wurden Passagen weggelassen, die die gesamte narrative Ebenenstruktur nicht wesentlich beeinflussen, entsprechend den von uns auf unserer Webseite vorgeschlagenen Ebenendefinitionen und unserem eigenen Urteil. 17 Texte wurden als Entwicklungskorpus zur Verfügung gestellt, das von den Teilnehmer`innen bei der Entwicklung von Richtlinien verwendet werden konnte. Tabelle 1 auf der nächsten Seite zeigt die Texte mit einigen Metadaten. Die Texte aus dem eigentlichen Annotationsexperiment sind in Tabelle 2 auf Seite $339 \mathrm{zu}$ finden. Alle Texte sind frei verfügbar und können über unser GitHub-Repository ${ }^{12}$ abgerufen werden.

Parallele Annotationen erstellen. Die Messung des IAA ist ein etablierter Weg, um Einblick in die intersubjektive Anwendbarkeit einer Annotationsrichtlinie zu gewinnen. Dazu müssen dieselben Texte von mehreren Personen unter Verwendung derselben Richtlinie annotiert werden.

Um dies im Shared Task umzusetzen, baten wir jede teilnehmende Gruppe, das Testkorpus mit Hilfe der Richtlinie eines anderen Teams zu annotieren. Darüber hinaus haben (bezahlte) studentische Hilfskräfte mit der gleichen Richtlinie annotiert. Damit wurde jede Richtlinie dreimal für denselben Text verwendet.

Workshop. Als Meilenstein im ersten Shared Task wurden alle Teilnehmer^innen zu einem Workshop eingeladen, der in Hamburg stattfand. Bis auf ein Team waren alle physisch anwesend. Die dreitägige Veranstaltung war wie folgt aufgebaut: Ziel des ersten Tages war es, dass alle Teilnehmer`innen die anderen Richtlinien besser verstehen. Dies wurde in Form von Kurzpräsentationen und einer Diskussion zur Identifizierung von Gemeinsamkeiten und Unterschieden realisiert. Am zweiten Tag wurden die Richtlinien detailliert ausgewertet. Am letzten Tag präsentierten die Organisator`innen die Bewertungsergebnisse sowie die Ergebnisse des IAA, worauf eine Gruppendiskussion der Ergebnisse und der nächsten Schritte den Workshop abschloss.

\subsubsection{Ausblick: Automatische Erkennung von Erzählebenen}

Der zweite Shared Task kann als ,normaler' NLP-Shared Task betrachtet werden und soll daher in erster Linie Forscher ${ }^{\star}$ innen in der maschinellen Sprachverar-

12 https://github.com/SharedTasksInTheDH (16.01.2020) 
Tab. 1: Übersicht über das Entwicklungskorpus

\begin{tabular}{|c|c|c|c|c|c|c|}
\hline Titel (orig.) & Autor & Titel (de) & Genre & Jahr & $\begin{array}{l}\text { Sprache } \\
\text { (orig.) }\end{array}$ & $\begin{array}{l}\text { Kom- } \\
\text { mentar }\end{array}$ \\
\hline & Äsop & $\begin{array}{l}\text { Der Wolf und } \\
\text { das Lamm }\end{array}$ & Märchen & $\begin{array}{l}600 \\
\text { v. Chr. }\end{array}$ & & \\
\hline & $\begin{array}{l}\text { Andersen, } \\
\text { Hans-Christian }\end{array}$ & Der Rosen-Elf & $\begin{array}{l}\text { Volks- } \\
\text { märchen }\end{array}$ & 1839 & $\mathrm{dk}$ & \\
\hline & $\begin{array}{l}\text { Andersen, } \\
\text { Hans-Christian }\end{array}$ & Das Liebespaar & $\begin{array}{l}\text { Volks- } \\
\text { märchen }\end{array}$ & 1862 & $\mathrm{dk}$ & \\
\hline $\begin{array}{l}\text { Se una notte } \\
\text { d'inverno un } \\
\text { viaggiatore }\end{array}$ & Calvino, Italo & $\begin{array}{l}\text { Wenn in einer } \\
\text { Winternacht } \\
\text { ein Reisender }\end{array}$ & Roman & 1979 & it & gekürzt \\
\hline Мститель & $\begin{array}{l}\text { Čechov, Anton } \\
\text { Pavlovič }\end{array}$ & Ein Rächer & $\begin{array}{l}\text { Kurzge- } \\
\text { schichte }\end{array}$ & 1887 & $\mathrm{ru}$ & \\
\hline $\begin{array}{l}\text { The Child's } \\
\text { story }\end{array}$ & $\begin{array}{l}\text { Dickens, } \\
\text { Charles }\end{array}$ & $\begin{array}{l}\text { Die Geschichte } \\
\text { des Kindes }\end{array}$ & $\begin{array}{l}\text { Kurzge- } \\
\text { schichte }\end{array}$ & 1852 & en & \\
\hline Die drei Federn & Grimm, Brüder & Die drei Federn & $\begin{array}{l}\text { Volks- } \\
\text { märchen }\end{array}$ & 1819 & de & \\
\hline $\begin{array}{l}\text { Das wohlfeile } \\
\text { Mittagessen }\end{array}$ & $\begin{array}{l}\text { Hebel, Johann } \\
\text { Peter }\end{array}$ & $\begin{array}{l}\text { Das wohlfeile } \\
\text { Mittagessen }\end{array}$ & Anekdote & 1804 & de & \\
\hline $\begin{array}{l}\text { Der geheilte } \\
\text { Patient }\end{array}$ & $\begin{array}{l}\text { Hebel, Johann } \\
\text { Peter }\end{array}$ & $\begin{array}{l}\text { Der geheilte } \\
\text { Patient }\end{array}$ & Anekdote & 1811 & de & \\
\hline $\begin{array}{l}\text { Hills Like White } \\
\text { Elephants }\end{array}$ & $\begin{array}{l}\text { Hemingway, } \\
\text { Ernest }\end{array}$ & $\begin{array}{l}\text { Hügel wie wei- } \\
\text { Be Elefanten }\end{array}$ & $\begin{array}{l}\text { Kurzge- } \\
\text { schichte }\end{array}$ & 1920 & en & \\
\hline $\begin{array}{l}\text { How the Leo- } \\
\text { pard got his } \\
\text { spots }\end{array}$ & $\begin{array}{l}\text { Kipling, } \\
\text { Rudyard }\end{array}$ & $\begin{array}{l}\text { Wie der Leo- } \\
\text { pard zu seinen } \\
\text { Flecken kam }\end{array}$ & $\begin{array}{l}\text { Kurzge- } \\
\text { schichte }\end{array}$ & 1901 & en & \\
\hline $\begin{array}{l}\text { Beyond the } \\
\text { Pale }\end{array}$ & $\begin{array}{l}\text { Kipling, } \\
\text { Rudyard }\end{array}$ & $\begin{array}{l}\text { Jenseits des } \\
\text { Blassen }\end{array}$ & $\begin{array}{l}\text { Kurzge- } \\
\text { schichte }\end{array}$ & 1888 & en & \\
\hline \multirow[t]{2}{*}{$\begin{array}{l}\text { Unwahrschein- } \\
\text { liche Wahrhaf- } \\
\text { tigkeiten }\end{array}$} & $\begin{array}{l}\text { Kleist, Heinrich } \\
\text { von }\end{array}$ & $\begin{array}{l}\text { Unwahrschein- } \\
\text { liche Wahrhaf- } \\
\text { tigkeiten }\end{array}$ & Anekdote & 1810 & de & \\
\hline & Lagerlöf, Selma & $\begin{array}{l}\text { Unter den } \\
\text { Kletterrosen }\end{array}$ & Erzählung & 1894 & sv & \\
\hline $\begin{array}{l}\text { The Cask of } \\
\text { Amontillado }\end{array}$ & $\begin{array}{l}\text { Poe, Edgar } \\
\text { Allen }\end{array}$ & $\begin{array}{l}\text { Das Fass des } \\
\text { Amontillado }\end{array}$ & $\begin{array}{l}\text { Kurzge- } \\
\text { schichte }\end{array}$ & 1846 & en & \\
\hline $\begin{array}{l}\text { Frankenstein } \\
\text { or The modern } \\
\text { Prometheus }\end{array}$ & Shelley, Mary & $\begin{array}{l}\text { Frankenstein } \\
\text { oder Der moder- } \\
\text { ne Prometheus }\end{array}$ & Roman & 1818 & en & gekürzt \\
\hline $\begin{array}{l}\text { A Haunted } \\
\text { House }\end{array}$ & Woolf, Virginia & Ein Spukhaus & $\begin{array}{l}\text { Kurzge- } \\
\text { schichte }\end{array}$ & 1921 & en & \\
\hline
\end{tabular}


Tab. 2: Übersicht über das Testkorpus

\begin{tabular}{|c|c|c|c|c|c|c|}
\hline Titel (orig.) & Autor & Titel (de) & Genre & Jahr & $\begin{array}{l}\text { Sprache } \\
\text { (orig.) }\end{array}$ & $\begin{array}{l}\text { Kom- } \\
\text { mentar }\end{array}$ \\
\hline Lenz & Büchner, Georg & Lenz & Novelle & 1839 & de & gekürzt \\
\hline $\begin{array}{l}\text { Выигрышный } \\
\text { билет }\end{array}$ & $\begin{array}{l}\text { Čechov, Anton } \\
\text { Pavlovič }\end{array}$ & Das Lotterielos & $\begin{array}{l}\text { Kurzge- } \\
\text { schichte }\end{array}$ & 1887 & $\mathrm{ru}$ & \\
\hline $\begin{array}{l}\text { Das Geschenk } \\
\text { der Heiligen } \\
\text { Drei Könige }\end{array}$ & Henry, 0. & $\begin{array}{l}\text { Das Geschenk } \\
\text { der Heiligen } \\
\text { Drei Könige }\end{array}$ & $\begin{array}{l}\text { Kurzge- } \\
\text { schichte }\end{array}$ & 1905 & de & \\
\hline Kleine Fabel & Kafka, Franz & Kleine Fabel & Märchen & 1920 & de & \\
\hline $\begin{array}{l}\text { Die blonde } \\
\text { Eckbert }\end{array}$ & Tieck, Ludwig & $\begin{array}{l}\text { Der weiße } \\
\text { Egbert }\end{array}$ & $\begin{array}{l}\text { literari- } \\
\text { sches } \\
\text { Märchen }\end{array}$ & 1797 & de & gekürzt \\
\hline $\begin{array}{l}\text { Der Schimmel- } \\
\text { reiter }\end{array}$ & Storm, Theodor & $\begin{array}{l}\text { Der Reiter des } \\
\text { weißen Pferdes }\end{array}$ & Novelle & 1888 & de & gekürzt \\
\hline $\begin{array}{l}\text { Anekdote aus } \\
\text { dem letzten } \\
\text { preußischen } \\
\text { Kriege }\end{array}$ & $\begin{array}{l}\text { Kleist, Heinrich } \\
\text { von }\end{array}$ & $\begin{array}{l}\text { Anekdote aus } \\
\text { dem letzten } \\
\text { Preußenkrieg }\end{array}$ & Anekdote & 1810 & de & \\
\hline $\begin{array}{l}\text { Herr Arnes } \\
\text { penningar }\end{array}$ & Lagerlöf, Selma & Der Schatz & Erzählung & 1904 & sv & gekürzt \\
\hline
\end{tabular}

beitung anziehen. Das annotierte Korpus wird in Entwicklungs-, Trainings- und Testdatensätze unterteilt und den Teilnehmer*innen zu bestimmten Zeitpunkten zur Verfügung gestellt. Die abschließende Bewertung erfordert dann, dass die Teilnehmer^innen ihre Vorhersagen an die Organisator`innen übermitteln, die sie wiederum mit den manuellen Annotationen des Testsets vergleichen. Dieser Shared Task soll in der SemEval-Community ${ }^{13}$ organisiert werden, um eine ausreichend große Teilnehmerzahl zu erreichen. Die Teilnehmer^innen müssen nicht mit Literaturwissenschaft, Narratologie oder digitalen Geisteswissenschaften vertraut oder darin erfahren sein, da die Aufgabe und ihre Schwierigkeiten in den Annotationen bereits kodiert sind. Das Ergebnis des zweiten Shared Tasks wird ein Vergleich von automatischen Systemen sein, die Erzählebenen erkennen.

13 SemEval hat sich als eine Art „Dach-Organisation“ für verschiedene Shared Tasks etabliert. Im Jahr 2019 z. B. wurden zwölf einzelne SemEval-Shared Tasks durchgeführt, von sprachübergreifendem semantischem Parsing (Task 1) bis hin zur Beantwortung von mathematischen Fragen (Task 10): http://alt.qcri.org/semeval2019/ (16.01.2020) 


\section{Zur Bewertung von Annotationsrichtlinien}

Die Bewertung von Annotationsrichtlinien ist ein neues Unterfangen, weshalb wir erst ein geeignetes Bewertungssystem etablieren mussten. Auch wenn dieses vermutlich nicht universell gültig oder anwendbar ist, glauben wir, dass der Ansatz zur Bewertung von Richtlinien für ähnliche Situationen relevant ist und an Projekte angepasst werden kann, die andere Präferenzen und Prioritäten haben könnten.

\subsection{Vorüberlegungen}

Unser Ziel ist es, Anforderungen und Prinzipien aus den Geisteswissenschaften sowie aus der Computerlinguistik/NLP zu berücksichtigen. Welche Bewertungsmethode wir auch immer am Ende anwenden würden, es mussten vier grundlegende Anforderungen erfüllt werden:

1. Ranking: Die Methode muss es ermöglichen, die Richtlinien auf einer Rangliste zu bewerten. Diese Rangliste muss so klar wie möglich sein und Mehrdeutigkeiten vermeiden.

2. Definiert und explizit: Shared Tasks sind ein Wettbewerb, bei dem die Einreichungen nach einer Zielfunktion geordnet werden. Diese Zielfunktion muss im Voraus und so genau wie möglich definiert werden, damit die Teilnehmer^innen wissen, worauf sie sich einlassen, und damit wenig Spielraum zur Anfechtung dieser Bewertung bleibt.

3. Praktikabel: Die Bewertung sollte innerhalb bestimmter praktischer Grenzen durchführbar sein. Konkret strebten wir eine Bewertungsmethode an, die im Rahmen eines zweitägigen Workshops durchgeführt werden konnte.

4. Bewertungskriterien: Die Bewertungsmethode muss unsere Bewertungsstandards widerspiegeln, d. h. wenn eine Richtlinie Aspekte enthält, die positiv bewertet werden, sollte diese Richtlinie höher eingestuft werden als eine Richtlinie ohne solche Aspekte. Die Definition von positiven/negativen Bewertungskriterien war eine Entscheidung, die die Organisator`innen treffen mussten.

Diese Anforderungen sind eine Folge des Ziels, Annotationsrichtlinien in einem Shared Task zu erstellen. Bei Shared Tasks in der natürlichen Sprachverarbeitung geht es darum, den Goldstandard so genau wie möglich zu reproduzieren, der dann je nach Aufgabe (Genauigkeit, f-Score, MUC-Score, etc.) unterschiedlich gemessen werden kann. Aber es gibt keine ,Grundwahrheit' für Annotationsrichtlinien. Selbst die Messung des IAA wäre keine optimale Zielfunktion, da es in den Daten Fälle geben kann, in denen unterschiedliche Textlesungen möglich sind, die sich aus 


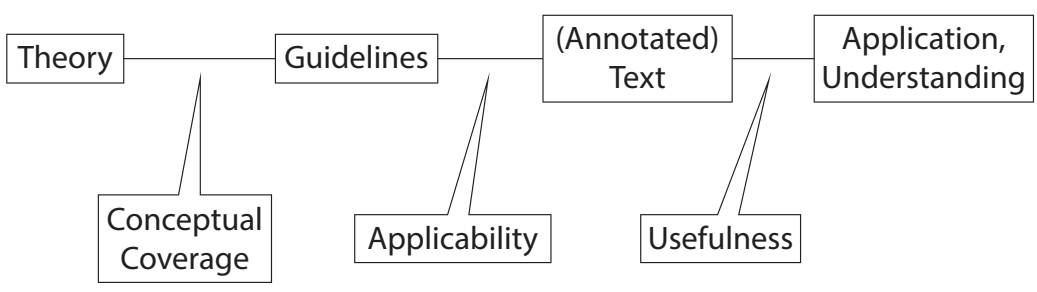

Abb. 2: Die drei Evaluationsdimensionen, die Forschungsgebiete in den digitalen Geisteswissenschaften verbinden.

einer Polyvalenz des Textes ergeben. In solchen Fällen würden Meinungsverschiedenheiten zwischen den Annotator*innen nicht auf einen Fehler in der Richtlinie hindeuten.

\subsection{Das Bewertungsmodell}

Die Bewertung wurde in drei verschiedenen Dimensionen durchgeführt: konzeptuelle Abdeckung, Anwendbarkeit und Nützlichkeit. Abbildung 2 zeigt schematisch, wo sich die Bewertungsdimensionen in Bezug auf Forschungsaktivitäten in den digitalen Geisteswissenschaften befinden. Die Abbildung projiziert die Dimensionen auf den Verlauf des gesamten Arbeitsprozesses, von der narratologischen Theorie über die Richtlinienerstellung bis hin zu annotierten Texten, und schließlich auf die Erkenntnisse, die sich aus der Anwendung der Richtlinie auf einzelne literarische Texte (manuelle Anwendung) oder ganze Korpora (automatische Erkennung) gewinnen lassen.

Die Dimension der konzeptuellen Abdeckung spiegelt wider, wie viel von einer theoretischen Grundlage durch eine Annotationsrichtlinie abgedeckt wird. Wenn eine Richtlinie explizit auf einer narratologischen Theorie basiert, könnte sie darauf abzielen, jede Definition, Regel und Ausnahme der Theorie vollständig umzusetzen. Eine andere Richtlinie, die auf der gleichen Theorie basiert, könnte einige Definitionen weglassen oder andere hinzufügen. Diese Dimension befindet sich auf der theoretischen Ebene und verbindet die Richtlinien mit der Theorie.

Die Anwendbarkeit setzt die Richtlinie in Beziehung zum Text und spiegelt wider, wie gut die Richtlinie die Annotator ${ }^{\star}$ innen auf die eigentlichen Annotationen vorbereitet, d. h. wie gut die Richtlinie angewendet werden kann. Die Anwendbarkeit einer Richtlinie kann beispielsweise durch durchdachte Beispiele, eine klare Struktur und/oder einen sorgfältigen Gebrauch der Terminologie erhöht werden. Die Dimension der Anwendbarkeit umfasst auch die erreichte Kohärenz und Systematik in den Annotationen. 
Schließlich bezieht sich die Dimension der Nützlichkeit auf die Anwendungsmöglichkeiten und das Verständnis des annotierten Textes: ,Anwendungsmöglichkeit' umfasst in diesem Fall sowohl nachfolgende Analyseschritte als auch Korpusanalysen, während ,Verständnis‘ sich auf eine hermeneutische Interpretation des Textes bezieht, die die Annotationen berücksichtigt. Unter der Annahme, dass Korpora gemäß der Richtlinie annotiert werden (entweder manuell oder, im Falle großer Korpora, automatisch), spiegelt diese Dimension wider, wie aufschlussreich die damit entstehenden Annotationen sind, d. h. wie ,viel‘ Einblick sie erlauben. Der Nutzen dient der Bewertung der Erkenntnisse, die durch die Untersuchung eines annotierten Textes oder Korpus gewonnen werden.

Die drei Dimensionen ermöglichen eine ausgewogene Bewertung von Richtlinien mit unterschiedlichen fachlichen und wissenschaftlichen Hintergründen, Zielen und Verständnissen narratologischer Konzepte. Wenn man sich nur auf eine der Dimensionen konzentriert, wird die Punktzahl in mindestens einer anderen verringert: Ein Leitfaden, der sich ausschließlich mit narratologischer Theorie befasst, könnte in der ersten Dimension eine hohe Punktzahl erreichen, wird aber in der zweiten Dimension schlecht abschneiden, da reine Theorie nicht gut anwendbar ist. Die Optimierung der Anwendbarkeit könnte zu Richtlinien führen, die alles oder nichts als erzählerische Ebene definieren und somit nicht sehr nützlich sind. Schließlich wird die blinde Optimierung des Nutzens zu Richtlinien führen, die nichts mit der narratologischen Theorie zu tun haben. Die Herausforderung, die dieser Shared Task für die Teilnehmer^innen darstellt, besteht also darin, ein Gleichgewicht zwischen den drei Dimensionen herzustellen.

Es ist anzunehmen, dass eine Annotationsrichtlinie in der Regel nicht alle drei Dimensionen abdecken muss, um eine nützliche Richtlinie für einen bestimmten Zweck zu sein. Richtlinien, die beispielsweise losgelöst oder völlig unabhängig von einem theoretischen Konzept sind, könnten trotzdem ein relevantes Thema ansprechen. Ebenso ist es nicht immer notwendig, sich mit Anwendungen und Zielen auseinanderzusetzen, d. h. mit dem Nutzen einer Richtlinie. Da Richtlinien und/oder Annotationen auch ein hervorragendes Werkzeug zur Textanalyse sind, kann ihre Erstellung ein ausreichendes Forschungsziel sein.

\subsection{Implementierung: Ein Fragebogen für die drei Dimensionen}

Um das dreidimensionale Bewertungsmodell zu implementieren, haben wir jede Dimension mit einer Reihe von spezifischen Fragen verknüpft, die für jede Richtlinie zu beantworten sind. Die Fragen stellen verschiedene Aspekte jeder Dimension dar und sollten für eine Richtlinie direkt beantwortbar sein. Abschnitt 4 listet jede Frage mit einer kurzen Beschreibung auf. 
Die Fragen wurden den Teilnehmer*innen zur Verfügung gestellt, bevor sie ihre Richtlinien eingereicht haben. In der Auswertung wurden sie auf zwei Arten verwendet: Erstens lieferten sie einen Leitfaden für die qualitative Bewertung. Durch die Befolgung des Online-Fragebogens und die Diskussion jeder Frage für jede Richtlinie haben wir sichergestellt, dass bei der Beurteilung für jede Richtlinie dieselben Kriterien angewendet werden und dass dieselben Aspekte in der Diskussion behandelt werden. Dies ist wichtig, um sowohl Fairness als auch Kohärenz bei der Bewertung zu gewährleisten. Die Diskussion war recht umfangreich und daher schwer zu dokumentieren, aber alle Teams bezeichneten sie als sehr hilfreich. Aus der Diskussion ergaben sich eine Reihe von Richtlinienverbesserungen, die im zweiten Band der Cultural Analytics-Sonderausgabe (Gius et al. 2019) dokumentiert werden.

Zweitens wurden die Fragen quantitativ beantwortet. Jede Frage wurde auf einer 4-Punkte-Likert-Skala bewertet, d.h. die Teilnehmer^innen wurden gebeten, für jede Richtlinie in jeder Frage Punkte zu vergeben, wobei eine hohe Punktzahl wünschenswert war. Wenn also Richtlinie A eine höhere Punktzahl als Richtlinie B hat, gilt sie als die bessere Richtlinie.

Unsere Bewertung definierte vier Fragen für die Dimensionen der konzeptuellen Abdeckung und Nützlichkeit und zwei Fragen für die Dimension der Anwendbarkeit. Um die Dimensionen gleich zu gewichten, wurden zwei weitere Bewertungen zur Anwendbarkeit durch das in das Intervall $[1 ; 4]$ skalierte IAA berechnet. Am Ende erhielt jede Richtlinie in jeder Dimension vier Punkte, die zunächst nach Dimension und dann zu einer Gesamtpunktzahl addiert wurden. Jedes Team bewertete alle anderen Richtlinien, was zu sieben Bewertungen pro Frage pro Richtlinie führte.

\section{Fragebogen}

\subsection{Konzeptionelle Abdeckung}

1. Ist das Konzept der Erzählebene explizit beschrieben?

Erklärung: Narrative Ebenen können beschrieben oder definiert werden. Dies hängt von der verwendeten Narratologie ab; einige von ihnen sind strukturalistisch, andere poststrukturalistisch. Unabhängig vom Modus, ist die Beschreibung/Definition verständlich und klar?

1: Ich habe nicht verstanden, was die Richtlinie als „erzählerische Ebene“ beschreibt. 
4: Ich habe das in der Richtlinie beschriebene Konzept vollständig verstanden.

2. Basiert das Konzept der Erzählebene auf bestehenden Konzepten?

Erklärung: Die Stufenkonzepte können selbst entworfen, an bestehenden Narratologien orientiert oder aus einer bestehenden Stufendefinition kopiert werden.

1: Die theoretische Beziehung des verwendeten Stufenkonzepts ist nicht klar.

4: Es wird deutlich erwähnt, ob das Stufenkonzept ausgearbeitet ist oder (teilweise) auf einer Theorie basiert.

3. Wie umfassend ist die Annotationsrichtlinie in Bezug auf Aspekte der Theorie? Lässt es etwas aus?

Erklärung: Wenn die Richtlinie auf einer Theorie oder mehreren Theorien basiert, beinhaltet sie dann die gesamte Theorie oder nur Teile davon? Gibt es Gründe, warum Aspekte ein- oder ausgeschlossen werden?

1: Die Richtlinie gibt die Ausweitung ihrer Abhängigkeit von der/den Theorie/n nicht klar an.

4: Die Richtlinie gibt den Umfang ihrer Theorienabhängigkeit unmissverständlich an.

4. Wie angemessen wird das Konzept der Erzählebene durch diese Richtlinie in Bezug auf die Erzählebenen umgesetzt?

Erklärung: Narratologien unterscheiden sich in ihrer Komplexität. Zuerst müssen Sie entscheiden, ob Komplexität oder Einfachheit (in Bezug auf x) wünschenswert ist, dann müssen Sie antworten:

1: Die Annotationsrichtlinie ist $\mathrm{zu}$ einfach oder $\mathrm{zu}$ komplex für narrative Ebenen und damit nicht ausreichend.

4: Die Komplexität der Richtlinie ist ausreichend.

\subsubsection{Anwendbarkeit}

1. Wie einfach ist es, den Leitfaden für Forscher^innen mit narratologischem Hintergrund anzuwenden?

Erklärung: Die Frage stellt eine Bewertung der Benutzerfreundlichkeit der Richtlinie für eine ${ }^{\star} n$ Annotator`in mit narratologischem Hintergrund dar. Indikatoren können sein: Komplexität der Konzepte, Länge des Leitfadens, 
Klarheit der Beispiele, klare Struktur, Schwierigkeit, Sonderfälle zu finden, etc.

1: Selbst als Narratologie-Expert*in musste ich den Leitfaden mehrmals lesen und/oder zusätzliche Literatur lesen.

4: Die Richtlinie ist sehr einfach anzuwenden, und ich wusste immer, was zu tun war.

2. Wie einfach ist es, den Leitfaden für Forscher^innen ohne narratologischen Hintergrund anzuwenden?

Erklärung: Die Frage stellt eine Bewertung der Benutzerfreundlichkeit der Richtlinie dar, wenn wir von jemandem ausgehen, der keinen narratologischen Hintergrund hat (z. B. Bachelor-Studierende). Indikatoren können sein: Komplexität der Konzepte, Länge des Leitfadens, Verwendung der Terminologie, Klarheit der Beispiele, Bezugnahme auf Beispiele nur durch Zitat, klare Struktur, Schwierigkeit, Sonderfälle $\mathrm{zu}$ finden, etc.

1: Nicht-Expert*innen haben keine Chance, diese Richtlinie zu nutzen.

4: Die Richtlinie ist sehr einfach anzuwenden und kann von Nicht-Expert*innen sofort angewendet werden.

3. Inter-Annotator-Übereinstimmung: Gamma-Scores (siehe unten)

4. Inter-Annotator-Übereinstimmung: Gamma-Scores (siehe unten)

\subsubsection{Nützlichkeit}

1. Gedankenexperiment: Unter der Annahme, dass die in der Annotationsrichtlinie definierten Erzählebenen automatisch auf einem riesigen Korpus erkannt werden können. Wie hilfreich sind diese Erzählebenen für eine interessante Korpusanalyse?

Erklärung: Diese Frage konzentriert sich auf die Relevanz der Annotationen auf Erzählebene für die textuelle Analyse großer Textmengen, z. B. für die Analyse von zeitlichen Entwicklungen in Bezug auf Erzählebenen oder eine genrebezogene Klassifizierung von Texten auf der Grundlage von Erzählebenen.

1: Die Annotationen der Erzählebenen sind für die Korpusanalyse irrelevant.

4: Die Annotationen liefern interessante Daten für die Korpusanalyse. 
2. Wie hilfreich sind sie als Basis für nachfolgende Korpus- oder einzelne Textanalyseschritte (die von Erzählebenen abhängen)?

Erklärung: Die Analyse einiger anderer textueller Phänomene hängt von den Erzählebenen ab, z. B. sollte die Chronologie innerhalb jeder Erzählebene analysiert werden, bevor sie für den gesamten Text analysiert wird. Diese Frage zielt darauf ab, ob die Analyse solcher Phänomene möglich oder sogar besser ist, wenn sie auf den Annotationen der Erzählebenen basiert.

1: Die Verwendung der Annotationen der Erzählebenen macht für nachfolgende Analysen keinen Unterschied.

4: Nachträgliche Analysen sind nur aufgrund der Annotationen der Erzählebenen möglich.

3. Gewinnen Sie durch die Anwendung der fremden Richtlinie neue Erkenntnisse über die Erzählebenen in Texten im Vergleich zur Anwendung Ihrer eigenen Richtlinie?

Erklärung: In den meisten Fällen ändert sich durch die Annotation eines Textes nach einer Richtlinie die Bewertung von textlichen Phänomenen im Text, z. B. die Qualität (oder Quantität) von Erzählebenen im Text.

1: Es macht keinen Unterschied - ich bekomme keine zusätzlichen Erkenntnisse mit der fremden Richtlinie.

4: Ich bekomme viele neue Erkenntnisse über die Erzählebenen in Texten, die auf dieser Richtlinie basieren.

4. Beeinflusst die Anwendung dieser Richtlinie Ihre Interpretation eines Textes? Erklärung: Interpretationen basieren in der Regel auf der Analyse eines Textes und damit auf der Beobachtung der Anwesenheit (oder Abwesenheit) bestimmter textueller Phänomene. Daher kann die Anwendung der Richtlinien zu Annotationen führen, die für Ihre Interpretation relevant sind, z. B. kann die Erkennung einer Erzählebene eines bestimmten Typs Ihre Interpretation der Zuverlässigkeit eines Erzählers beeinflussen.

1: Meine Interpretation ist unabhängig von den Annotationen, die auf der Richtlinie basieren.

4: Meine Interpretation basiert in erster Linie auf den Annotationen, die auf der Richtlinie basieren.

\subsection{Messung der Inter-Annotator-Übereinstimmung}

Bei diesem Shared Task haben wir die von Yann Mathet, Antoine Widlöcher und Jean-Philippe Métivier (2015, 437-479) entwickelte Metrik y (gamma) verwendet. Ihr 
Endergebnis kombiniert beobachtete Unterschiede mit zufälligen Unterschieden ( $\mathrm{y}$ wird also anhand von Unterschieden berechnet, während die meisten Kennzahlen anhand von Gemeinsamkeiten berechnet werden). Dies geschieht, um Bewertungsschemata mit unterschiedlicher Komplexität vergleichen zu können und um zu vermeiden, dass einfachere Schemata bevorzugt werden (wenn das Schema einfacher ist, ist die zufällige Übereinstimmung höher).

Um Gamma zu messen, verwendeten wir eine Implementierung, die von den Entwicklern auf ihrer Webseite zur Verfügung gestellt wurde. Die Art und Weise, wie dort erwartete Meinungsverschiedenheiten berechnet werden, führt aber zu Problemen, wenn nur wenige Annotationen vorhanden sind. Wenn eine einzelne Annotation den gesamten Text abdeckt, was für Annotationen von Erzählebenen durchaus plausibel ist, gibt es keine Möglichkeit, den Text zu teilen und die Annotationen neu zu mischen. Um dies zu umgehen, haben wir Gamma sowohl für jeden Text einzeln berechnet als auch für alle acht Texte zusammen. Die letztgenannte Punktzahl wurde dann für die endgültige Bewertung verwendet.

\subsection{Integration der Bewertungsscores}

Die Endnote für jede Richtlinie wurde wie folgt berechnet:

1. Für jede der zehn Fragen wird das arithmetische Mittel über alle Antworten berechnet. Daraus ergeben sich zehn Werte, verteilt auf drei Dimensionen: vier Fragen/Werte in der ersten Dimension, zwei Fragen/Werte in der zweiten und vier Fragen/Werte in der dritten.

2. Die Gamma-Scores werden auf das Intervall von $[0 ; 1]$ und als zwei ,virtuelle Fragen“ in der zweiten Dimension hinzugefügt. Daraus ergeben sich vier Werte pro Dimension, jeweils im Intervall $[0 ; 1]$.

3. In jeder Dimension werden alle vier (Mittel-)Werte addiert.

4. Daraus ergibt sich eine Punktzahl für jede Dimension, sodass die Richtlinien entsprechend geordnet werden können. Als Gesamtnote haben wir die Summe der Ergebnisse in allen Dimensionen berechnet.

Damit ergeben sich die Gesamtergebnisse, die in Tabelle 3 auf der nächsten Seite dargestellt sind. Die Gewinner-Guideline ist demnach Guideline V. ${ }^{14}$ Was auffällt ist, dass die Richtlinie gar nicht in allen Kategorien Höchstpunktzahlen erzielen konnte, dafür aber eine gute Balance zwischen den Dimensionen gefunden hat.

14 Für diese - und alle anderen - Guidelines vgl. Gius, Reiter und Willand (2019). 
Tab. 3: Finales Ranking der Guidelines

\begin{tabular}{lrrrrr}
\hline Guideline & Dimension & \multicolumn{3}{c}{} & Overall \\
\hline & $\begin{array}{c}\text { Conceptual } \\
\text { Coverage }\end{array}$ & $\begin{array}{l}\text { Applicability } \\
\text { Punkte }\end{array}$ & Gamma & Usefulness & \\
\hline Guideline V & 14.14 & 12.09 & 0.25 & $\mathbf{1 2 . 8 8}$ & $\mathbf{3 9 . 1 0}$ \\
Guideline II & 11.17 & 11.89 & 0.24 & 12.57 & 35.63 \\
Guideline VI & 12.33 & 11.01 & 0.21 & 11.37 & 34.71 \\
Guideline IV & $\mathbf{1 4 . 4 3}$ & 7.71 & 0.05 & 11.26 & 33.40 \\
Guideline VIII & 8.10 & $\mathbf{1 4 . 1 4}$ & 0.30 & 9.12 & 31.36 \\
Guideline VII & 11.60 & 9.82 & 0.23 & 9.77 & 31.18 \\
Guideline I & 7.83 & 10.39 & 0.18 & 10.00 & 28.22 \\
Guideline III & 10.29 & 6.48 & 0.07 & 10.95 & 27.72 \\
\hline
\end{tabular}

\section{Fazit}

Zum jetzigen Zeitpunkt (November 2019) befindet sich der erste Shared Task in den Digital Humanities, der sich die Erstellung von Annotationsrichtlinien für das narratologische Phänomen der Erzählebene zur Aufgabe gemacht hat, noch in der Arbeitsphase, für ein endgültiges Fazit ist es daher zu früh. Ein Zwischenfazit kann jedoch durchaus gezogen werden:

Die Erstellung von Annotationsrichtlinien ist kein Selbstläufer. Es handelt sich gerade nicht um eine ,Reformatierung ' von existierendem Wissen, das nur in anderer Art und Weise (etwa formalisiert) aufgeschrieben wird. Die systematische Annotation führt regelmäßig dazu, dass neue (Teil-)Phänomene narratologischer Phänomene entdeckt oder alte neu gesehen werden können. Dies ist ein direktes Ergebnis der systematischen Anwendung der Richtlinien durch mehrere Annotierende. Dabei werden die Annotierenden (und damit die Richtlinien) mit Problemen konfrontiert, die sonst womöglich unbeachtet geblieben wären. Umso wichtiger ist es, geeignete Formate zu etablieren, die Anreize schaffen, Annotationsrichtlinien zu erstellen oder weiterzuentwickeln. Shared Tasks in den Digital Literary Studies sind also als Innovationstreiber zu verstehen, und zwar sowohl in technischer wie auch in konzeptueller Hinsicht. 


\section{Literatur}

Artstein, Ron und Massimo Poesio. Inter-Coder Agreement for Computational Linguistics. In: Computer Linguistics 34, no. 4. Cambridge, MA: Massachusetts Institute of Technology Press. 2008, S. 555-596.

Cohen, Jacob. A Coefficient of Agreement for Nominal Scales. In: Educational and Psychological Measurement 20, no. 1. Thousand Oaks, CA: SAGE Publishing. 1960, S. 37-46.

Fleiss, Joseph L. Measuring Nominal Scale Agreement Among Many Raters. In: Psychological Bulletin 76, no. 5. Washington, DC: American Psychological Association. 1971, S. 420-428. DOI: $10.1037 / \mathrm{h} 0031619$.

Fournier, Chris. Evaluating Text Segmentation Using Boundary Edit Distance. Bd. 1: Long Papers. In: Proceedings of the 51st Annual Meeting of the Association for Computational Linguistics (Volume 1: Long Paper). Sofia, Bulgarien: Association for Computational Linguistics. 2013, S. 1702-1712. URL: https://www.aclweb.org/anthology/P13-1167/ (16.01.2020).

Gius, Evelyn und Janina Jacke. The Hermeneutic Profit of Annotation: On Preventing and Fostering Disagreement in Literary Analysis. In: International Journal of Humanities and Arts Computing 11, no. 2. Edinburgh: Edinburgh University Press. Oktober 2017, S. 233-254. DOI: 10.3366/ijhac.2017.0194.

Gius, Evelyn, Nils Reiter und Marcus Willand. A Shared Task for the Digital Humanities Chapter 2: Evaluating Annotation Guidelines. In: Journal of Cultural Analytics. November 2019. DOI: 10.22148/16.049.

Jannidis, Fotis, Hubertus Kohle und Malte Rehbein. Digitale Geisteswissenschaften. Eine Einführung. Stuttgart: Metzler. 2017.

Jockers, Matthew Lee. Text Analysis with R for Students of Literature. Cham: Springer. 2014.

Mathet, Yann, Antoine Widlöcher und Jean-Philippe Métivier. The Unified and Holistic Method Gamma (y) for Inter-Annotator Agreement Measure and Alignment. In: Computational Linguistics 41, no. 3. Cambridge, MA: Massachusetts Institute of Technology Press. 2015, S. 437-479.

Pagel, Janis, Nils Reiter, Ina Rösinger und Sarah Schulz. A Unified Text Annotation Workflow for Diverse Goals. In: Sandra Kübler und Heike Zinsmeister (Hrsg.), Proceedings of the Workshop on Annotation in Digital Humanities, co-located with ESSLLI 2018. Sofia, Bulgarien. 2018. URL: http://ceur-ws.org/Vol-2155/pagel.pdf (16.01.2020).

Parra Escartín, Carla, Wessel Reijers, Teresa Lynn, Joss Moorkens, Andy Way und Chao-Hong Liu. Ethical Considerations in NLP Shared Tasks. In: Proceedings of the First ACL Workshop on Ethics in Natural Language Processing. Valencia: Association for Computational Linguistics. 2017, S. 66-73. URL: https://www.aclweb.org/anthology/W17-1608/ (16.01.2020).

Ruder, Sebastian. URL:https://nlpprogress.com (17.01.2019).

Schreibman, Susan, Ray Siemens und John Unsworth (Hrsg.). A Companion to Digital Humanities. Oxford: Blackwell Publishing. 2004. URL: http://www.digitalhumanities.org/companion/ (16.01.2020).

Shared Tasks in the Digital Humanities. URL: https://github.com/SharedTasksInTheDH (16.01.2020).

Shared Tasks in the Digital Humanities. Narrative Levels. URL: https://sharedtasksinthedh. github.io/levels/ (16.01.2020).

Siemens, Ray und Susan Schreibman. A Companion to Digital Literary Studies. Oxford: WileyBlackwell. 2008. 
Silva Knuppel, Anandi und Maria José Afanador-Llach (Hrsg.). The Programming Historian. URL: https://programminghistorian.org (16.01.2020).

Stubbs, Amber und James Pustejovsky. Natural Language Annotation for Machine Learning. Sebastopol, CA: O’Reilly Media, Inc. 2013, S. $23 \mathrm{ff}$.

TEl. 2019. URL: http://www.tei-c.org (16.01.2020).

Witten, Ian H. und Frank Eibe. Data Mining Practical Machine Learning Tools and Techniques, 2. San Francisco, CA: Morgan Kaufmann Publisher, Elsevier. 2005, S. 144 ff. 